Marquette University

e-Publications@Marquette

College of Nursing Faculty Research and

Publications

Nursing, College of

$12-1-2017$

\title{
The Positive Thinking Skills Scale: A Screening Measure for Early Identification of Depressive Thoughts
}

Abir K. Bekhet

Marquette University, abir.bekhet@marquette.edu

Mauricio Garnier-Villarreal

Marquette University, mauricio.garniervillarreal@marquette.edu

Accepted version. Applied Nursing Research, Vol. 38 (December 2017): 5-8. DOI. (C) 2017 Elsevier B.V. Used with permission. 


\section{Marquette University \\ e-Publications@Marquette}

\section{Nursing Faculty Research and Publications/College of Nursing}

This paper is NOT THE PUBLISHED VERSION; but the author's final, peer-reviewed manuscript. The published version may be accessed by following the link in the citation

below.

Journal, Vol. xx, No. xx (xxxx): xx-xx. DOI. This article is ( $[$ [Publisher] and permission has been granted for this version to appear in e-Publications@Marquette. [Publisher] does not grant permission for this article to be further copied/distributed or hosted elsewhere without the express permission from [Publisher].

\section{The Positive Thinking Skills Scale: A screening measure for early identification of depressive thoughts}

Abir K. Bekhet

Marquette University College of Nursing, Milwaukee, WI

Mauricio Garnier-Villarreal

Marquette University College of Nursing, Milwaukee, WI

\section{Abstract}

Background

Depression is currently considered the second leading cause of disability worldwide. Positive thinking is a cognitive process that helps individuals to deal with problems more effectively, and has been suggested as a useful strategy for coping with adversity, including depression. The Positive Thinking Skills Scale (PTSS) is a reliable and valid measure that captures the frequency of use of positive thinking skills that can help in the early identification of the possibility of developing depressive thoughts. However, no meaningful cutoff score has been established for the PTSS. 
Aim

To establish a cutoff score for the PTSS for early identification of risk for depression.

\section{Methods}

This study used a receiver operating characteristic (ROC) curve to establish a PTSS cutoff score for risk for depression, using the Center for Epidemiological Studies-Depression Scale (CES-D) as the gold standard measure.

\section{Results}

In a sample of 109 caregivers, the ROC showed that the cutoff score of PTSS that best classify the participants is 13.5 . With this PTSS score, $77.8 \%$ of the subjects with low CES-D are classify correctly, and $69.6 \%$ of the subjects with high CES-D are classify correctly. Since the PTSS score should be integer numbers, functionally the cutoff would be 13.

\section{Conclusion}

The study showed that a cut off score of 13 is a point at which referral, intervention, or treatment would be recommended. Consequently, this can help in the early identification of depressive symptoms that might develop because of the stress of caregiving.

\section{Keywords}

Positive thinking; Depression; Caregivers

Depression is currently considered the second leading cause of disability worldwide as compared to it being the fourth leading cause of disability in 1990 and the third leading cause of disability in 2000 (Ferrari et al., 2013). In 2014, it was estimated that 6.7\% (16 million American adults) of those aged 18 or older had at least one major depressive episode (National Institute for Mental Health, 2015). Depression is a serious and debilitating mental illness that affects people of all ages however; it is as twice likely to occur in females more than males (Kessler, 2003). Depression has a substantial impact on work productivity and it is associated with increasing morbidity and mortality as well as has a significant impact on the global burden and the health care costs (Furlanetto et al., 2005, Yeung et al., 2007, Zauszniewski and Bekhet, 2012).

Positive thinking is a cognitive process that helps individuals to deal with problems more effectively, and has been proved to be a valuable strategy for dealing with hardship, including depression (Tod et al., 2011, Bekhet and Zauszniewski, 2013). Positive thinking has been found to be associated with less depression, better quality of life, less burden, greater life satisfaction, better psychological and physical well-being (Appold, 2009, Bekhet et al., 2012, Bekhet and Zauszniewski, 2013, Dekker et al., 2009, Jung et al., 2007, Lightsey and Boyraz, 2011, Zauszniewski et al., 2009). In addition, positive thinking was found to have mediating 
effects on the relationship between caregiver's depression and their children's challenging behaviors (Bekhet, 2016).

Findings of a recent study indicated that high depressive cognitions in Autism Spectrum Disorder (ASD) caregivers were associated with lower positive thinking $(r=-0.39 ; p<0.001)$ (Bekhet, 2017). Therefore, early recognition of the positive thinking skills used by caregivers can help early detection of the possibility of developing depressive thoughts which is vital in preventing the development of clinical depression and suicide (Sousa et al., 2005, Bekhet and Zauszniewski, 2013). As suggested by Beck's cognitive theory of depression, the depressive thoughts, or in other words the automatic negative thoughts, precede the development of clinical depression (Beck, Brown, Steer, Eidelson, \& Riskind, 1987). Therefore, early identification of the negative automatic thoughts is essential in preventing clinical depression and the costs associated with it. In 2013, in response to the need of positive thinking training intervention for caregivers and the lack of a direct measure of positive thinking intervention fidelity, a new eight-item Positive Thinking Skills Scale (PTSS), which measures the frequency with which intervention recipients use positive thinking skills, was developed (Bekhet \& Zauszniewski, 2013). The PTSS is a reliable and valid measure that capture the frequency of use of positive thinking skills that will help in the early detection of the possibility of developing depressive thoughts and prevention of depressive symptoms that might develop because of caregiving. Thus, the purpose of this study was to determine a meaningful cutoff score on the PTSS, using a receiver operating characteristic (ROC) curve, to identify the point at which referral, intervention, or treatment would be recommended.

\section{The Positive Thinking Skills Scale}

The PTSS is an eight item skills scale with responses on a 4-point Likert scale, ranging from $0=$ never to $3=$ always. Scores may range from 0 to 24 ; the higher the scores, the higher the frequency of using the skills constitute positive thinking. Examples of the scale items are: "Transform negative thoughts into positive thoughts" and "Highlight positive aspects of the situation." The PTSS is reliable as indicated by a Chronbach's alpha of $0.90(\alpha=0.90)$. Criterion validity was supported by significant correlations $(p<0.01)$ with positive cognitions $(r=0.53)$, resourcefulness $(r=0.63)$, depression $(r=-0.45)$, and general well-being $(r=0.40)$ (Bekhet and Zauszniewski, 2013, Bekhet, 2017). The PTSS is different from the other measures in that it is a brief measure that takes only $5 \mathrm{~min}$ to complete and is a direct measure of intervention fidelity to skills that may be taught to caregivers during a positive thinking training. This measure can be used for assessing the skills used by caregivers and can be used to evaluate the frequency of using them.

The PTSS has been translated into Turkish (Akin, Uysal, \& Akin, 2015). Results of confirmatory factor analysis in the Turkish language demonstrated that the eight items loaded on one factor in a sample of 295 university students. Internal consistency coefficient of the scale was 0.87 and the corrected item-total correlations ranged from 0.54 to 0.68 . These results demonstrate that this scale is a valid and reliable instrument. Another study conducted by Tully (2016) aimed at examining the effects of positive thinking on the perception of practice environment stressors in hospital based US nurses. The results indicated that the PTSS is a reliable scale as indicated by a Cronbach's alpha of 0.89 in sample of 128 acute care nurses. 


\section{Method}

\subsection{Design}

The study was a secondary analysis of data from a cross sectional study of 109 caregivers of persons with autism spectrum disorders (ASD). The original study used a cross-sectional descriptive design to assess the psychometric properties of the PTSS.

\subsection{Sample, setting, and data collection}

In the parent study, the sample included 109 caregivers of persons with ASD who were able to read and understand English, had Internet access, and resided in the United States. No potential participants were excluded based on gender, race, or socioeconomic status. Participants were recruited by convenience sampling from the Interactive ASD Network (IAN) Research registry service. The institutional review board (IRB) approval was obtained from the university. IAN contacted caregivers by email and sent them an IRB-approved flyer directing them to the Internet website (www.surveymonkey.com) where they accessed the consent form and a link to the study questionnaires. Participants provided their email addresses at the end of the survey to claim their incentives and they were sent a code that could be redeemed for US\$15 at www.amazon.com (Bekhet \& Zauszniewski, 2013).

\subsection{Instruments}

Descriptive data on caregivers of persons with ASD were collected. In addition, measures of positive thinking and depression were collected.

Depression was measured by the CES-D (Radloff, 1977). The CES-D is a 20-item Likert-type scale ranging from $0=$ rarely or none of the time to $3=$ most or all of the time; it was designed originally to assess depressive symptoms in adults and proved to be acceptable to both general and clinical populations (Radloff, 1977). Participants are asked to indicate how frequently they experience depressive feelings and behaviors during the past week. Scores may range from 0 to 60 , after reverse coding 4 items, with higher scores indicating the presence of more depressive symptoms.

The CES-D has reported internal consistency with a Cronbach's alpha of 0.92 in mothers of children with ASD (Ekas, Whitman, \& Shivers, 2009). Criterion validity of the CES-D was supported by significant correlations in the expected direction with the Hamilton Clinician's Rating Scale and with the Raskin Rating Scale (Radloff, 1977).

\subsection{Analysis}

Data were analyzed using R 3.3.3 (R Core Team, 2017). Descriptive statistics are presented along with measures of reliability for the scales. The reliability was evaluated with the Maximal Reliability (MR) coefficient. MR estimates the reliability of a scale assuming items have a different weight into it. MR is the maximal possible reliability for a linear combination of the scale items. MR involves the estimation of the optimal linear combination (OLC), which are the 
weights for each item, OLC represents the different item weights for MR. MR measures reliability of a scale, unlike Cronbach alpha which estimate inter item correlation (Raykov, 2012, Li, 1997). MR is estimated with the R package semTools (semTools Contributors, 2017).

The CES-D was the criteria to classify participants as those with and without relevant clinical depressive symptoms. For the CES-D a score of 16 is used to differentiate relevant clinical depressive symptoms (Radloff, 2007).

A receiver operating characteristic curve analysis $(R O C)$ is used to identify the probability of proper classification based of different scores of the positive thinking scale (PT). The ROC determines the ability of the PT to differentiate between participants with and without relevant clinical depressive symptoms based of the CES-D classification.

The ROC analysis estimates the sensitivity and sensibility of classification for different scores of PT, sensitivity is the probability that PT model classify $\hat{y}=1$ when $y=1$ is present in our observations (true positives); an sensibility is the probability that our model classify $\hat{y}=0$ when $y=0$ in present in our observations (true negatives). From these estimates the optimal cutoff score is chosen in function of a desire balance between them. The ROC gives an estimate of area under the curve (AUC) which represents an overall performance of the PT score to classify participants correctly, a $1.0(100 \%)$ indicates that PT classify subjects with perfect accuracy, the accuracy of the AUC has been evaluated as 0.50 to $0.70=$ low; 0.70 to $0.90=$ moderate; and $>0.90=$ high $($ Streiner \& Cairney, 2007).

\section{Results}

The sample consists of 109 participants, $96.3 \%$ are female, $88.1 \%$ are Caucasian, $10.1 \%$ are Hispanic, and 1.8 are African American; the average age in the sample was $42.02(S D=7.0)$. Table 1 shows the descriptive statistics for the PTSS and CES-D scale scores. The Positive Thinking scale showed high reliability with an MR $=0.92$, while the CES-D scale presented a high reliability with an MR $=0.95$. The PT and CES-D scales presents a moderate negative correlation $(r=-0.45, p<0.001)$, as PT increases participants depressive symptoms decreases, for 1 unit increase in PT the depressive symptoms decreases 0.45 units.

Table 1. Descriptive statistics.

\begin{tabular}{|ccccccc|}
\hline Scale & Mean & SD & Median & Minimum & Maximum \\
Positive Thinking Skills & 14.28 & 4.94 & 15 & 2 & 24 \\
CES-D & 17.77 & 12.21 & 14 & 2 & 54 \\
\hline
\end{tabular}

For this sample $57.8 \%$ (63) participants presented a CES-D score lower than 16 . When looking at PT mean differences between participants with high and low CESD-D (LD, HD), the LD participants present a lower mean $(15.9, S D=4.3)$ than the HD participants $(11.9, S D=4.9)$, we have evidence that this difference is not $0(\mathrm{t}(89.3)=-4.48, p<0.001)$. This mean difference shows a Cohen's $d=0.89(95 \% \mathrm{Cl}=-1.30,-0.48)$, which is consider a large effect (Cohen, 1992), showing that the group means are 0.89 standard deviations apart. 
The ROC analysis was used to determine the optimum cutoff score for PT associated with the highest possible combination of sensibility and sensitivity. On how the data was specified, the model looks at the ability to properly classify participants with CES-D lower than 16 . The optimal cutoff would have a high sensitivity and sensibility, while keeping them in balance, it would not be optimal for one of them to be high while the other is low. For the PT, the ROC shows a AUC $=0.75(95 \% \mathrm{Cl}=0.65,0.85)$, this model presents a moderate ability to properly classify the participants based on their clinically relevant depressive symptoms (Fig. 1).

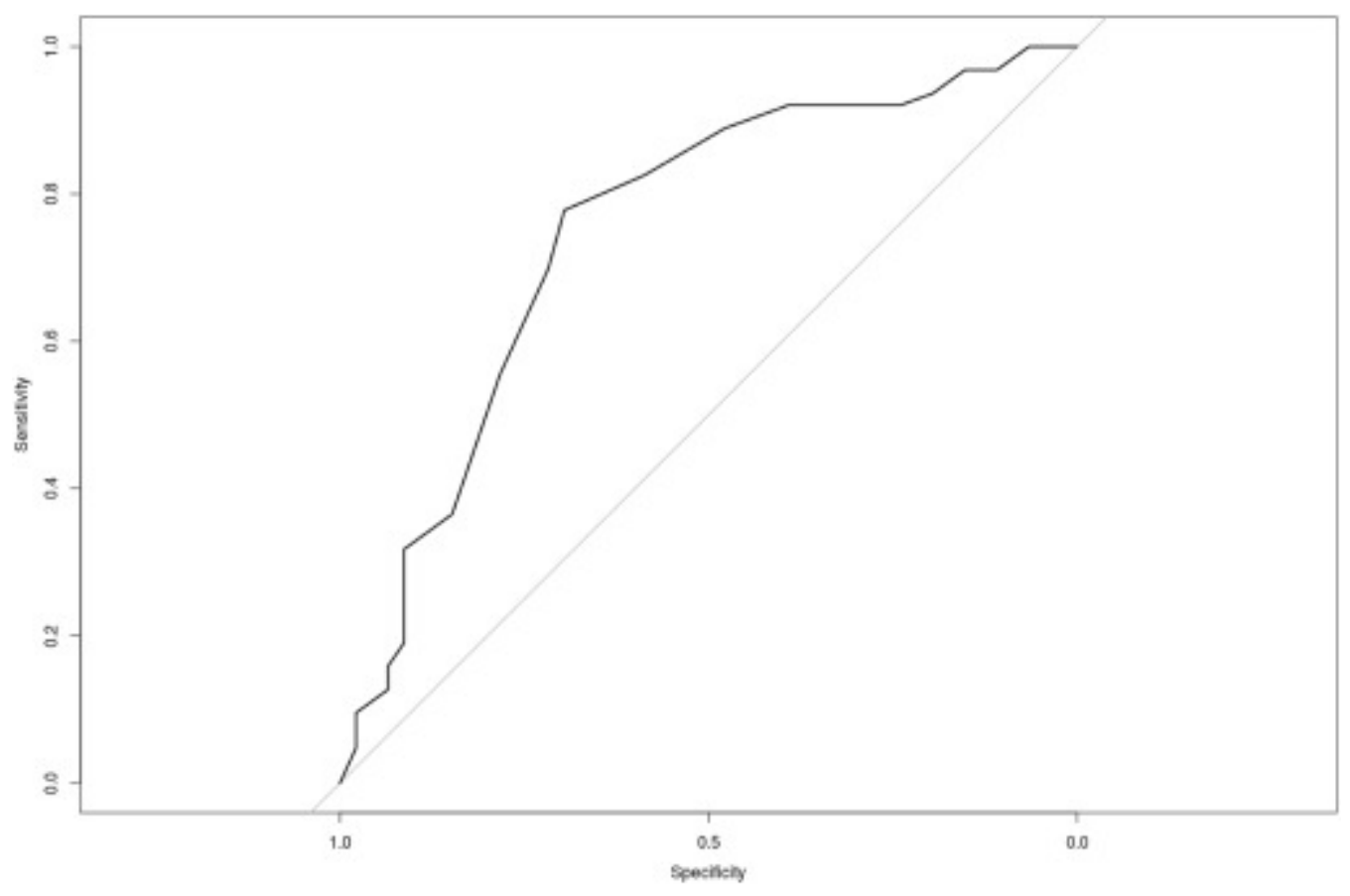

Fig. 1. ROC analysis.

Looking at the sensitivity and specificity (Table 2), the cutoff score of PT that best classify the participants is 13.5 . With this PT score, $77.8 \%$ of the subjects with low CES-D are classify correctly, and $69.6 \%$ of the subjects with high CES-D are classify correctly. Since the PT score is on integer numbers, functionally the cutoff would be 13 .

Table 2. Analysis of scale parameters for possible cutoff scores across different Positive Thinking Scale scores.

\begin{tabular}{|cccc|}
\hline Sensitivity & & Specificity & Cutoff \\
$\mathbf{0 . 8 8 9}$ & 0.478 & 11.5 & 12.5 \\
$\mathbf{0 . 8 2 5}$ & 0.587 & 13.5 \\
$\mathbf{0 . 7 7 8}$ & 0.696 & 14.5 \\
$\mathbf{0 . 6 9 8}$ & 0.717 & 15.5 \\
$\mathbf{0 . 5 5 6}$ & 0.783 & 16.5 \\
$\mathbf{0 . 3 6 5}$ & 0.848 & \\
\hline
\end{tabular}


For this sample, with the cutoff of 13, we have 71 subjects with high PTSS, and 38 with low PTSS. Comparing to the classification made by the CES-D, PTSS classify 52 subjects as true positives, meaning they had a CES-D lower than 16 and PTSS higher than 13. PTSS classify correctly 27 subjects as true negatives, meaning they had a CES-D higher than 16 and a PTSS lower 13. Classify as false positives 19 subjects, they had CES-D higher than 16 and a PTSS higher than 13. And finally, classify as false negatives 11 subjects, they had CES-D lower than 16 and a PTSS lower than 13.

\subsection{Discussion and implications for practice}

This study represents a first attempt to establish a cutoff score on the Positive Thinking Skills Scale (PTSS). Cutoff score on the PTSS is essential to identify risk for clinical depression and the point at which referral, intervention, or treatment would be recommended. The PTSS scale can be used by nurses and health care professionals to plan for primary and secondary prevention strategies to help caregivers to retain, attain, or maintain their psychological wellbeing. Primary prevention is directed toward enhancing caregivers 'wellness by preventing or decreasing the stress and burden of caregivers, especially those who are at high risk of developing stress and depression. Those could be caregivers who have recently learned about their child diagnosis. Our results provide empirical data that can enhance the assessment of early identification of depressive thoughts across a range of caregivers of persons with autism with various ages, educational background, as well as different ethnicities. More specifically, the ages of caregivers in this study ranged from 24 to 58 years and the sample included Caucasian, African American, and Hispanic, as well as those with a wide range of education.

The PTSS will help the health care providers to identify which skill/skills out of the eight positive thinking skills are used by caregivers so these skills can be reinforced and which ones are not in use so that they need to be taught for better outcomes for ASD caregivers. The PTSS can be used on a secondary level prevention. For example, for those caregivers who are diagnosed with depression, the positive thinking skills can be taught to them and then the scale can be administered to identify which skills are used by them and how frequent they are using them. Because the scale consists of only eight items, it can be administered frequently, and it can help in assessing the progress in using these skills.

Identification of a meaningful cutoff score on the PTSS can help early recognition of depressive thoughts and may lead to a brief, more tailored psychotherapy, thus helping in decreasing the treatment costs.

The internal consistency of the PTSS in the study reported here was 0.90 , somewhat higher than those previously reported for acute care nurses in USA (Tully, 2016) and university students in Turkey (Akin, Uysal, \& Akin, 2015), this might be due to the greater diversity in this Internet-based national sample. In order to determine the cutoff score on the PTSS, we used the AUC, which generated a moderate level of accuracy. The PTSS discriminated $77.8 \%$ of individuals with and without depressive thoughts, which is considered a good level of accuracy. This is, in fact, similar to the results of a previous research by Zauszniewski and Bekhet (2012) who used AUC to determine the cut off scores on the Depressive Cognition Scale and they found that AUC discriminated between participants with and without serious depressive 
thinking in $80 \%$ of cases and they concluded that it represents a moderate level of accuracy. This study supports the cut score of 13 as optimal. Sensitivity is higher at this score, which is important in minimizing the risk of false negatives (Uslu et al., 2008). Additional research is needed to address the ability of the PTSS to detect negative thinking that might precede the development of clinical depression at this cutoff score using a larger sample size. Future studies may also investigate whether the cutoff score found with this sample of caregivers of persons with ASD is applicable to people in other countries and ethnicities. Translation and validation of the PTSS to different languages will provide the means for the health care professionals and clinicians with a valid and reliable means of assessing the frequency of using the positive thinking skills and assessing the negative thoughts that might precede the development of clinical depression. Cross-cultural comparisons of the cutoff scores for PTSS among Americans and people from various other cultures for which the instrument has been translated and validated, such as the Turkish populations is recommended (Akin et al., 2015).

\section{Acknowledgement}

The parent study was funded by Way Klinger Young Scholar Award awarded to Dr. Abir K. Bekhet.

\section{References}

1 A. Akin, R. Uysal, U. AkinThe validity and reliability of Turkish version of the Positive

Thinking Skills Scale Journal of European Education, 5 (2) (2015), pp. 16-22

$2 \mathrm{~K}$. AppoldOvercoming challenges: The power of positive thinking 72-73, American

Medical Technologists Events (2009), p. 108 Retrieved from

http://www.highbeam.com/doc/1G1-229543473.html

3 A.T. Beck, G. Brown, R.A. Steer, J.I. Eidelson, J.H. RiskindDifferentiating anxiety and depression: A test of the cognitive content specificity hypothesis Journal of Abnormal Psychology, 96 (1987), pp. 179-183

4 A.K. BekhetThe Mediating Effects of Positive Cognitions on Autism Caregivers' depression and their Children Challenging Behaviors Archives of Psychiatric Nursing, 30 (2016), pp. 13-18

$5 \mathrm{~A}$. BekhetPositive thinking training intervention for caregivers of persons with autism: Establishing fidelity Archives of Psychiatric Nursing http://dx.doi.org/10.1016/j.apnu.2017.02.006 (2017)

6 A. Bekhet, J. ZauszniewskiMeasuring use of Positive Thinking Skills Scale: Psychometric testing of a new scale Western Journal of Nursing Research, 35 (8) (2013), pp. 1074-1093

7 A. Bekhet, N. Johnson, J.A. ZauszniewskiResilience among family members of persons with autism Issues in Mental Health Nursing, 33 (10) (2012), pp. 650-656

8 J. CohenA power primer Psychological Bulletin, 112 (1992), pp. 155-159

9 R. Core TeamR: A language and environment for statistical computing R Foundation for Statistical Computing, Vienna, Austria (2017) https://www.R-project.org/ 
10 R.L. Dekker, A.R. Peden, T.A. Lennie, M.P. Schooler, D.K. MoserLiving with depressive symptoms: Patients with heart failure American Journal of Critical Care, 18 (2009), pp. 310-318

11 N.V. Ekas, T.L. Whitman, C. ShiversReligiosity, spirituality, and socioemotional functioning in mothers of children with autism spectrum disorder Journal of Autism and Developmental Disorders, 39 (5) (2009), pp. 706-719

12 A.J. Ferrari, F.J. Charlson, R.E. Norman, S.B. Patten, G. Freedman, C.L. Murray, ,.., H.A. WhitefordBurden of depressive disorders by country, sex, age, and year: Findings from the Global Burden of Disease Study 2010 PIOS (2013) Retrieved from http://dx.doi.org/10.1371/journal.med.1001547

13 M.L. Furlanetto, M.V. Mendlowicz, J. Romildo BuenoThe validity of the Beck Depression Inventory-Short Form as a screening and diagnostic instrument for moderate and severe depression in medical inpatients Journal of Affective Disorders, 86 (2005), pp. 87-91

14 J.Y. Jung, Y.H. Oh, K.S. Oh, D.W. Suh, Y.C. Shin, H.J. KimPositive thinking and life satisfaction amongst Koreans Yonsei Medical Journal, 48 (2007), pp. 371-378

15 R.C. KesslerEpidemiology of women and depression Journal of Affective Disorders, 74 (2003), pp. 5-13

$16 \mathrm{H}$. LiA unifying expression for the maximal reliability of a linear composite Psychometrika, 62 (1997), pp. 245-2

17 R.O. Lightsey, G. BoyrazDo positive thinking and meaning mediate the positive affectlife satisfaction relationship? Canadian Journal of Behavioral Science, 43 (2011), pp. 203-213

18 National Institute for Mental Health, 2015 National Institute for Mental Health Retrieved on April 1st, 2017 from https://www.nimh.nih.gov/health/statistics/prevalence/majordepression-among-adults.shtml (2015)

19 L.S. RadloffThe -ES-D scale: A self-report depression scale for research in the general population Applied Psychological Measurement, 1 (1977), pp. 385-401

$20 \mathrm{~T}$. RaykovScale construction and development using structural equation modeling R.H. Hoyle (Ed.), Handbook of structural equation modeling, Guilford, New York (2012), pp. $472-494$

21 semTools ContributorssemTools: Useful tools for structural equation modeling $\mathrm{R}$ package version 0.4-15.903 (2017) Retrieved from https://CRAN.Rproject.org/package $=$ semTools

22 V.D. Sousa, J.A. Zauszniewski, I.A. Mendes, M.L. ZanettiCross-cultural equivalence and psychometric properties of the Portuguese version of the Depressive Cognition Scale Journal of Nursin Measurement, 13 (2) (2005), pp. 87-99

23 D.L. Streiner, J. CairneyWhat's under the ROC? An introduction to receiver operating characteristics curves Canadian Journal of Psychiatry, 52 (2007), pp. 121-128

$24 \mathrm{~A}$. Tod, C. Warnock, P. AllmarkA critique of positive thinking for patients with cancer Nursing Standard, 25 (2011), pp. 43-47

$25 \mathrm{~S}$. TullyPositive thinking and the perception of work-related stress in acute care nurses (2016) Unpublished dissertation 
26 R.I. Uslu, E.G. Kapci, B. Oncu, M. Ugurlu, H. TurkcaparPsychometric properties and cutoff scores of the Beck Depression Inventory-II in Turkish adolescents Journal of Clinical Psychology in Medical Settings, 15 (2008), pp. 225-233

27 A. Yeung, K.M. Overstreet, E.V. AlbertCurrent practices in depression care Journal of Continuing Education in the Health Professions, 27 (Suppl. 1) (2007), pp. S9-S17

28 J.A. Zauszniewski, A. BekhetDeveloping a screening measure for early detection of depressive symptoms: The Depressive Cognition Scale Western Journal of Nursing Research, 34 (2) (2012), pp. 228-242

29 J.A. Zauszniewski, A.K. Bekhet, M.J. SureskyEffects on resilience qualities of women family caregivers of adults with mental illness: The role of positive cognitions Archives of Psychiatric Nursing, 23 (2009), pp. 412-422

30 L.S. RadloffCenter for epidemiological studies-Depressed Mood Scale

$31 \mathrm{~J}$. Fischer, K. Corcoron (Eds.), Measures for clinical practice: A sourcebook, Vol. 2, Oxford University Press, New York, NY (2007), pp. 139-141 Adults 